\title{
Incontinência urinária e sua relação com variáveis obstétricas
}

\section{Urinary incontinence and its relationship with obstetric variables}

\author{
Jaíza Marques Medeiros e Silva, Maria do Socorro Barbosa e Silva
}

Como citar este artigo: SILVA, J. M. M.; SILVA, M.S.B. Incontinência urinária e sua relação com variáveis obstétricas. Revista Saúde (Sta. Maria). 2019; 45 (2).

\section{Autor correspondente:}

Nome: Jaíza Marques Medeiros e Silva

E-mail: jaizamarques_@hotmail.com Telefone: ( 83 ) 988308235

Formação Profissional:

Mestranda em Saúde Pública pela Universidade Estadual da Paraiba (UEPB) Formada em

Fisioterapia pela UEPB, que fica na cidade de Campina Grande. Paraíba, Brasil.

Filiação Institucional: Universidade Estadual da Paraiba

Endereço para correspondência:

Rua: Rodrigues Alves $n^{\circ}: 1380$

Bairro: Boa vista Cidade: Campina Grande Estado: Paraíba CEP: 58428-795

Data de Submissão:

08/03/20 18

Data de aceite:

26/05/2019

Conflito de Interesse: Não há conflito de interesse

\section{(cc) $\mathrm{BY}-\mathrm{NC}-\mathrm{ND}$}

\section{RESUMO}

O estudo objetivou determinar a prevalência da incontinência urinária (IU) na gestação e identificar sua associação com variáveis obstétricas. Estudo transversal em que foram incluídas gestantes com rotina de pré-natal, na faixa etária de 18 e 35 anos, com gestação de baixo risco e no segundo ou terceiro trimestre. Foram utilizados o questionário sociodemográfico e o International Consultation on Incontinence Questionnaire - Short Form. As gestantes foram agrupadas de acordo com a presença ou não de sintomas de perda urinária. Os testes estatísticos utilizados foram o Teste de Fisher e MannWhitney. Prevalência de IU foi 36,7\%. Na análise bivariada, não foi encontrada nenhuma associação significativa entre as variáveis obstétricas e a IU, porém na análise das variáveis contínuas, houve associação significativa entre paridade e IU. É necessária atuação dos profissionais da saúde no combate a IU no período gestacional, identificando os possíveis fatores de risco para o aparecimento dessa patologia.

PALAVRAS-CHAVE: Incontinência urinária; Gestação; Gestantes.

\section{ABSTRACT}

The study aimed to determine the prevalence of urinary incontinence (UI) during pregnancy and to identify its association with obstetric variables. A cross-sectional study in which pregnant women with prenatal routine, in the age group of 18 and 35 years old, with low-risk gestation and in the second or third trimester were included. The sociodemographic questionnaire and the International Consultation on Incontinence Questionnaire - Short Form were used. The pregnant women were grouped according to the presence or absence of symptoms of urinary loss. The statistical tests used were Fisher's test and Mann-Whitney test. Prevalence of UI was $36.7 \%$. In the bivariate analysis, no significant association was found between the obstetric variables and the $\mathrm{UI}$, but in the analysis of the continuous variables, there was a significant association between parity and UI. It is necessary the act of the health professionals in the fight against $\mathrm{UI}$ in the gestational period, identifying the possible risk factors for the appearance of this pathology.

KEYWORDS: Urinary incontinence; Gestation; Pregnant women. 


\section{INTRODUÇÃO}

A gravidez é associada a um período de felicidade e de grandes mudanças para a maioria das mulheres. Infelizmente, a gestação pode ser também considerada um período de risco para o desenvolvimento da disfunção do assoalho pélvico'.

As disfunções dos músculos do assoalho pélvico (DAPs) são consideradas condições ginecológicas que afetam a vida da mulher independente da sua idade. As DAPs possuem vários fatores etiológicos que podem culminar no aparecimento da incontinência urinária (IU), incontinência anal (IA), disfunções sexuais e prolapsos de órgãos pélvicos².

A incontinência urinária é definida como qualquer perda involuntária de urina e é considerada um relevante problema de saúde pública atualmente ${ }^{3}$. Nas mulheres, a incontinência apresenta grande impacto na qualidade de vida, interferindo também no intercurso sexual e nas atividades básicas da vida diária ${ }^{4}$. Uma revisão sistemática da literatura realizada com 22 artigos de estudos brasileiros identificou uma prevalência de IU variando de 5,8\% a 72\% ${ }^{5}$. No período gestacional, estudos brasileiros identificaram uma prevalência que variou de $19 \%^{6}$ a $71 \%{ }^{7}$, tornando a IU na gestação um importante tema para estudos.

Durante a gestação, ocorrem várias alterações a nível hormonal, estrutural e funcional como a dilatação uretral, compressão mecânica causada pelo crescimento uterino que, por sua vez, também ocasiona a redução da capacidade vesical, podem permitir o aparecimento ou não da incontinência urinária ${ }^{8}$. Além disso, o aumento da sobrecarga exercida no assoalho pélvico pelo aumento de peso durante a gestação pode ocasionar sintomas de perda urinária ${ }^{9}$.

Estudos internacionais vem apresentando fatores como idade materna ${ }^{10,11}$, idade gestacional ${ }^{11}$, paridade ${ }^{12,13} \mathrm{e}$ índice de massa corporal ${ }^{14}$ bem como sua classificação $0^{15}$ sendo associados à incontinência urinária. Porém são escassos poucos os estudos nacionais que abordam tais associações ${ }^{7,16-17}$.

Desse modo, o presente estudo buscou identificar a prevalência de incontinência urinária na gestação e avaliar a associação das variáveis obstétricas com esse tipo de disfunção dos músculos do assoalho pélvico. Espera-se que os dados gerados possam fornecer informações acerca dos fatores ligados à incontinência urinária, auxiliando no entendimento sobre o tema.

\section{MATERIAL E MÉTODOS}

Este estudo é do tipo transversal, com coleta de dados primários e é parte de um estudo mais amplo que objetivou realizar uma avaliação da função sexual e urinária em gestantes cadastradas na Estratégia Saúde da Família no município de Campina Grande-PB.

Foram incluídas 60 gestantes com rotina de pré-natal, ou seja, aquelas gestantes assistidas por médicos ou 
enfermeiros das unidades durante o período gestacional, com idade superior ou igual a 18 anos e inferior ou igual a 35 anos, com gestação de baixo risco e que estivesse no segundo ou terceiro trimestre gestacional. Foram excluídas gestantes que tivessem diabetes mellitus, litíase renal, infecção do trato urinário inferior (ITU), que fizessem uso de medicamentos que interferissem na função do trato urinário inferior (TUI) e que não tivessem vida sexual ativa nos últimos 30 dias. $\mathrm{O}$ trabalho de campo foi realizado por uma de entrevistadora, devidamente treinada.

A amostra da pesquisa foi por conveniência, ou seja, não probabilística, em que não foram utilizadas técnicas de amostragem. A variável dependente analisada foi a incontinência urinária e as variáveis independentes foram: idade da gestante, idade gestacional, trimestre gestacional, Índice de Massa Corporal (IMC), classificação do IMC, paridade, classificação da paridade. A classificação do IMC foi realizada através da classificação proposta por Atalah et.al (1997) ${ }^{18}$.

Os instrumentos utilizados para a realização da pesquisa foram: Questionário contendo variáveis sóciodemográficas como também variáveis obstétricas e o International Consultation on Incontinence Questionnaire - Short Form (ICIQ-SF).

Incontinência urinária

A prevalência de incontinência urinária foi avaliada através do ICIQ-SF que é um instrumento composto por quatro questões que analisam a frequência, a gravidade e o impacto da incontinência urinária, além de um conjunto de oito itens de auto-diagnóstico, sendo pautadas as causas e situações em que ocorrem incontinência ${ }^{19}$.

\section{Procedimentos estatísticos}

Os dados sócio-demográficos foram organizados através de frequências e porcentagens. As gestantes foram agrupadas em dois grupos de acordo com o auto-diagnóstico, ou seja, no G1 foram consideradas as gestantes que possuíam incontinência urinária e no G2 as gestantes que não possuíam incontinência continentes. A idade da gestante, a idade gestacional, a paridade e o índice de massa corporal são apresentados sob a forma de média e desvio-padrão (DP) e a significância estatística das diferenças de médias entre os grupos foi verificada por meio do teste Mann-Whitney por se tratar de amostras independentes. As variáveis categóricas foram avaliadas através do teste de Fisher. Em todas as análises foi adotado um intervalo de confiança de 95\%. As informações estatísticas foram obtidas com o auxílio do aplicativo estatístico Statistical Package for Social Sciences (SPSS) versão 22.0.

\section{Questões éticas}


A pesquisa maior da qual este estudo fez parte foi submetida e aprovada pelo Comitê de Ética em Pesquisa (CEP) da Universidade Estadual da Paraíba (UEPB) (CAAE: 55073116.3.0000.5187). As gestantes, ao aceitarem participar da pesquisa, assinaram o Termo de Consentimento Livre e Esclarecido, segundo Resolução 466/12 do Conselho Nacional de Saúde.

\section{RESULTADOS E DISCUSSÃO}

A International Continence Society $(\mathrm{ICS})^{20}$ define incontinência urinária como uma condição na qual ocorre queixa de qualquer perda de urina que aconteça de maneira involuntária. Assim, das 60 gestantes que participaram da pesquisa, $22(36,7 \%)$ possuíam sintomas de perda urinária na gestação. Essa prevalência é bem menor quando comparada ao estudo realizado em Portugal com 237 gestantes, em que 51,89\% relataram sintomas de perda urinária durante a gestação $0^{13}$, porém um estudo realizado na Etiópia com 422 mulheres grávidas também apresentou uma baixa prevalência de incontinência urinária nesse período $(11,4 \%)^{21}$.

A análise bivariada entre as variáveis obstétricas e a presença de sintomas de incontinência urinária está representada na tabela 1 . Entre as gestantes com sintomas de incontinência urinária, a maioria estava no terceiro trimestre gestacional $(20 \%)$, diferentemente das gestantes sem perda urinária, em que a maioria estava no segundo trimestre $(33,3 \%)$. Estudos internacionais também encontraram aumento da prevalência de incontinência urinária e sintomas urinários com a progressão da gestação ${ }^{21,22}$. Esse aumento na prevalência de incontinência no terceiro trimestre pode estar relacionada a alterações mecânicas como o efeito da pressão do útero gravídico sobre a bexiga, associado com 0 aumento de sua sensibilidade e redução significativa da capacidade vesical ${ }^{23}$.

Houve maior proporção de gestantes nulíparas no grupo de mulheres sem perda urinária (38,3\%), porém isso não ocorreu de maneira significativa ( $p=0,088)$. Estudo realizado com 750 gestantes apresentou associação significativa entre paridade e sintomas de incontinência urinária, expondo resultados em que gestantes multíparas estiveram mais associadas com o aparecimento de sintomas de incontinência urinária $(p=<0,001)^{11}$. Outros estudos também apresentaram associação significativa entre paridade e incontinência urinária ${ }^{10,12}$.

Em relação à classificação do IMC, houve uma maior proporção de mulheres incontinentes com sobrepeso $(18,3)$ e de mulheres continentes com peso normal (30), porém não houve associação estatisticamente positiva entre classificação do IMC e a incontinência urinária $(p=0,251)$. Esse dado corrobora com estudo realizado com 250 gestantes que teve como objetivo identificar os fatores de risco para incontinência urinária na gestação e não houve associação entre classificação do IMC e IU $(p=0,631)^{12}$. Porém, estudo nacional realizado nas cidades de Santos/SP, Pirassununga/SP e Curitiba/PR apresenta associação estatisticamente significativa entre gestantes consideradas obesas e o aparecimento 
de incontinência urinária ${ }^{7}$. Estudo realizado na Turquia com 287 mulheres grávidas também evidenciou associação estatisticamente significativa entre classificação do IMC e incontinência urinária $(p=0,030)^{24}$.

Tabela 1 - Distribuição das gestantes estudadas de acordo com a associação entre variáveis obstétricas categóricas e incontinência urinária (\%). Campina Grande-PB, Brasil.

\begin{tabular}{llcc} 
& \multicolumn{3}{c}{ Presença de incontinência urinária } \\
\hline Variáveis categóricas & Sim & Não & p \\
\hline Trimestre gestacional & & & 0,789 \\
Segundo trimestre & 16,7 & 33,3 & \\
Terceiro trimestre & 20,0 & 30,0 & \\
Classificação da paridade & & & 0,088 \\
Nulípara & 11,7 & 38,3 & \\
Primípara & 16,7 & 16,7 & \\
Multípara & 8,3 & 8,3 & \\
Classificação do IMC & & & \\
Baixo peso & 3,3 & 3,3 & \\
Normal & 8,3 & 30,0 & \\
Sobrepeso & 18,3 & 23,4 & \\
Obesidade & 6,7 & 6,7 & \\
\hline
\end{tabular}

Teste de Fischer

Na tabela 2 estão representadas as médias e desvios padrões das variáveis contínuas de idade, idade gestacional, IMC e paridade e sua associação com a incontinência urinária. Houve associação significativa entre a paridade e a presença de incontinência urinária. Esse dado corrobora com estudo realizado na Turquia com 132 mulheres grávidas que por meio da análise de regressão logística associou a paridade com a presença de incontinência urinária durante a gestação ${ }^{25}$. Porém em estudo realizado em Campo Grande no Brasil com 80 gestantes em uma maternidade, não foi encontrada associação entre incontinência urinária de estresse e paridade $(p=0,61)$, bem como entre urge-incontinência e paridade $(p=0,07)^{26}$.

Neste estudo não houve diferença significativa entre a idade materna nos dois grupos em relação à presença de sintomas de perda urinária, não demonstrando associação $(p=0,193)$. Esse dado não condiz com estudos internacionais e estudo nacional em que as gestantes que possuem idade mais avançada possuem associação significativa com a presença do quadro de incontinência urinária ${ }^{7,10,12}$. Porém estudo realizado com 500 gestantes na cidade de São José do Rio Preto também não apresentou diferença significativa entre as idades maternas ${ }^{27}$. 
Em relação à idade gestacional, neste estudo não houve associação significativa entre os grupos de gestantes. Esse dado não corrobora com estudo internacional realizado na Turquia em que foi encontrado diferença estatisticamente significativa entre as idades gestacionais entre os grupos de gestantes continentes e incontinentes $(p=<0,001)^{11}$. Outros estudos também apresentaram associação significativa entre idade gestacional e incontinência urinária ${ }^{7,23}$. Porém, pesquisa realizada com 250 gestantes corroborou com estudo em questão, pois também não apresentou diferença significa entre os grupos estudados ${ }^{12}$. Uma revisão de literatura apresenta que a IU aumenta consideravelmente com 0 aumento da idade gestacional, tendo uma variação de prevalência de $18,6 \%$ para $75 \%{ }^{28}$.

Neste estudo, o IMC não apresentou diferença significativa entre os grupos estudados ( $p=0,759)$, porém estudo realizado com 465 gestantes em duas maternidades do estado de São Paulo e duas no estado do Paraná apresentou diferença estatisticamente significativa entre os grupos $(p=0,01)^{7}$. 0 aumento da massa corporal, do útero gravídico e as alterações hormonais existente nesse período podem provocar o aumento do tônus muscular por tensão excessiva fazendo com que o músculo não resista a uma carga mínima devido a diminuição da atividade elétrica, dificultando a função de suporte e continência desses músculos ${ }^{9}$.

Tabela 2 - Médias e desvios-padrão (DP) dos valores da idade da gestante, idade gestacional, paridade e Índice de Massa Corporal (IMC) segundo grupos de acordo com a presença de incontinência urinária. Campina Grande, Paraíba, Brasil

Presença de incontinência urinária

\begin{tabular}{|c|c|c|c|c|c|c|c|}
\hline \multirow[t]{2}{*}{ Variáveis } & \multicolumn{3}{|c|}{ Sim } & \multicolumn{3}{|c|}{ Não } & \multirow[b]{2}{*}{$p$} \\
\hline & $\mathrm{n}$ & Média & DP & $\mathrm{n}$ & Média & $\mathrm{DP}$ & \\
\hline Idade da gestante & 22 & 25,00 & 4,50 & 48 & 23,39 & 3,40 & 0,193 \\
\hline Idade gestacional & 22 & 26,45 & 7,58 & 48 & 27,10 & 7,84 & 0,759 \\
\hline Paridade & 22 & 1,00 & 0,92 & 48 & 0,52 & 0,72 & $0,036^{*}$ \\
\hline IMC & 22 & 28,17 & 3,90 & 48 & 27,23 & 4,10 & 0,759 \\
\hline
\end{tabular}

*Diferença estatisticamente significativa entre os grupos (Teste Mann-Whitney) DP=Desvio padrão; $p<0,05$

\section{CONSIDERAÇÕES FINAIS}

Os resultados deste estudo sugerem uma provável influência da paridade no aparecimento de sintomas de perda urinária quando se compara as médias entre os grupos, porém quando se compara a freqüência essa influência deixa de existir. Com relação à análise bivariada, não foi encontrada associação de nenhuma variável obstétrica com a incontinência urinária. 
Novas investigações devem ser realizadas a fim de verificar a incontinência urinária em mulheres no período gestacional. Sugere-se que outros estudos também abordem outras variáveis obstétricas como o tipo de parto e o uso de instrumentalização para o parto vaginal e sua associação com os sintomas de incontinência urinária.

Este trabalho, além de ter como limitação a amostra de participantes, também apresenta limitações inerentes aos estudos transversais, pois embora tenha sido evidenciada associação entre paridade e incontinência urinária, não determina causas e efeitos entre variáveis obstétricas e a incontinência urinária. Com isso, sugere-se a realização de estudos longitudinais acerca da incontinência urinária e seus respectivos fatores relacionados às alterações gestacionais em uma amostra de gestantes representativa da população, permitindo a ampliação do conhecimento acerca do tema em questão.

O estudo, contudo, explicita a importância da atuação dos profissionais de saúde acerca do tema e a necessidade implantação de políticas públicas que gerem a promoção de saúde em relação à incontinência urinária neste público-alvo para que, assim, a gestante tenha uma melhor qualidade de vida. A fisioterapia é uma importante aliada na prevenção à incontinência urinária, pois, utiliza-se dos exercícios do assoalho pélvico, bem como técnicas de biofeedback para promover o melhor funcionamento dessa parte corporal da mulher em que poucas conhecem o seu significado e importância.

\section{REFERÊNCIAS}

1. Ashton-Miller JA, DeLancey JO. Functional anatomy of the female pelvic floor. Ann N Y Acad Sci. 2007; 1101: 266-296.

2. Almeida MBA, Barra AA, Figueiredo EM, Velloso FSB, Silva AL, Monteiro MVC, et.al. Disfunção de assoalho pélvico em atletas. FEMININA. 2011; 39 (8): 395-402.

3. Berlezi EM, Bem AD, Antonello C, Leite MT, Bertola EM. Incontinência urinária em mulheres no período pós-menopausa: um problema de saúde pública. Rev Bras Geriatr Gerontol. 2009; 12(2): 159-173.

4. Moccellin AS, Rett MT, Driusso P. Incontinência urinária na gestação: implicações na qualidade de vida. Rev Bras Saúde Matern Infant. 2014; 14(2): 147-154. 
5. Justina LBD. Prevalência de incontinência urinária feminina no Brasil: uma revisão sistemática. Revista Inspirar. 2013; 5(2):1-7.

6. Riesco MLG, Fernandes-Trevisan K, Leister N, Cruz CS, Caroci AS, Zanetti MRD. Incontinência urinária relacionada à força muscular perineal no primeiro trimestre da gestação: estudo transversa. Rev Esc Enferm USP. 2014; 48(Esp): 33-39.

7. Oliveira C, Seleme M, Cansi PF, Consentino RFDC, Kumakura FY, Moreira GA, et al. Urinary incontinence in pregnant women and its relation with sócio-demographic variables and quality of life. Rev Assoc Med Brasi. 2013; 59(5): 460-466.

8. Moises ECD, Brito LGD, Duarte G, Freitas MMS. Disfunções miccionais no período gestacional e puerperal. FEMININA. 2011; 39(8): 409-412.

9. Moccellin AS, Rett MT, Driusso P. Existe alteração na função dos músculos do assoalho pélvico e abdominais em primigestas no segundo e terceiro trimestre gestacional?. Fisioter Pesqui. 2016; 23(2):136-141.

10. Lin Kun-Ling, Shen Ching-Ju, Wu Ming-Ping, Long Cheng-Yu; Wu Chin-Hu, Wang Chiu-Lin. Comparison of Low Urinary Tract Symptoms during Pregnancy between Primiparous and Multiparous Women. Bio Med Research International. 2014; $1-5$.

11. Dinç A. Prevalence of Urinary Incontinence During Pregnancy and Associated Risk Factors. Low Urin Tract Symptoms. 2017. DOI: 10.1111/luts.12182

12. Balik G, Guven ESG, Tekin YB, Senturk S, Kagitci M, Usturne I, et.al. Lower Urinary Tract Symptoms and Urinary Incontinence During Pregnancy. Low Urin Tract Symptoms. 2014. DOI: 10.1111/luts.12082.

13. Rocha J, Brandão P, Melo A, Torres S, Mota L, Costa F. Avaliação da Incontinência Urinária na Gravidez e no Pós-Parto: Estudo Observacional. Acta Med Port. 2017;30(7-8):568-572. 
14. Liang Ching-Chung, Chang Shuenn-Dhy, Lin Shu-Jen, Lin Yu-Jr. Lower urinary tract symptoms in primiparous women before and during pregnancy. Arch Gynecol Obstet 2012; 285:1205-1210.

15. Daly D, Clarke M, Begley C. Urinary incontinence in nulliparous women before and during pregnancy: prevalence, incidence, type, and risk factors. Int Urogynecol J. 2018; 29(3): 353-362.

16. Sacomori C, Boer L, Sperandio FF, Cardoso FL. Prevalência e variáveis associadas à incontinência urinária no terceiro trimestre gestacional. Rev. Bras. Saúde Matern. Infant. 2013; 13 (3): 215-221.

17. Mathias AERA, Pitangui ACR, Freitas HGV, Arantes VA, Vilela FMF, Dias TG. Prevalência de incontinência urinária durante o terceiro trimestre gestacional. Arq. Ciênc. Saúde. 2014; 21(4) 101-5.

18. Atalah E, Castillo C, Castro R, Aldea A. Propuesta de un nuevo estándar de evaluación nutricional en embarazadas. Rev Méd Chile 1997; 125: 1429-36.

19. Tamanini JTN, Dambros M, D'Ancona CAL, Palma PCR, Netto Jr NR. Validação para português do "International Consultation on Incontinence Questionnaire - Short Form" (ICIQ-SF). Rev Saúde Pública. 2004; 38(3): 438-444.

20. Abrams P, Cardoso L, Fall M, Griffiths D, Rosier P, Ulmesten U, et al. The standardisation sub-committee of the International Continence Society. Urology. 2003;61(1):37-49.

21. Bekele A, Adefris M, Demeke S. Urinary incontinence among pregnant women, following antenatal care at University of Gondar Hospital, North West Ethiopia. BMC Pregnancy and Childbirth. 2016; 16:333.

22. Beksac AT, Aydin E, Orhan C, Karaagaoglu E, Akbayrak T. Gestational Urinary Incontinence in Nulliparous Pregnancy-A Pilot Study. Journal of Clinical and Diagnostic Research. 2017; 11(8): QC01-QC03.

23. Scarpa KP, Herrmann V, Palma PCR, Ricetto CLZ, Morais S. Sintomas do trato urinário inferior três anos após o parto: estudo prospectivo. Rev Bras Ginecol Obstet. 2008; 30 (7): 355-9. 
24. Kok G, Seven M, Guvenc G, Akyuz A. Urinary Incontinence in Pregnant Women: Prevalence, Associated Factors, and Its $E \llbracket$ ects on Health-Related Quality of Life J. Wound Ostomy Continence Nurs. 2016;43(5):511-516.

25. Dermican N, Özmen U, Köktürk F, Kücük H, Ata S, Harma M, et.al. What are the probable predictors of urinary incontinence during pregnancy? Peer J. 2016; 4:e2283. doi: 10.7717/peerj.2283.

26. Lima MC, Moccelin GBA, Silva MB, Nogueira GB. Effect of mode of delivery and parities on the occurrence of urinary incontinence during pregnancy. Fisioter. Mov. 2015; 28(1): 107-115.

27. Martins G, Soler ZASG, Cordeiro JA, Amaro JL, Moore KN. Prevalence and risk factors for urinary incontinence in healthy pregnant Brazilian women. Int Urogynecol J. 2010; 21:1271-1277.

28. Sangsawang B. Risk factors for the development of stress urinary incontinence during pregnancy in primigravidae: a review of the literature. European Journal of Obstetrics \& Gynecology and Reproductive Biology. 2014. http://dx.doi.org/10.1016/j.ejogrb.2014.04.010. 